\title{
RNAscope in situ hybridization-based method for detecting DUX4 RNA expression in vitro
}

\author{
GHOLAMHOSSEIN AMINI CHERMAHINI, ${ }^{1}$ AFROOZ RASHNONEJAD, ${ }^{1}$ and SCOTT Q. HARPER ${ }^{1,2}$ \\ ${ }^{1}$ Center for Gene Therapy, The Research Institute at Nationwide Children's Hospital, Columbus, Ohio 43205, USA \\ ${ }^{2}$ Department of Pediatrics, The Ohio State University, Columbus, Ohio 43205, USA
}

\begin{abstract}
Facioscapulohumeral muscular dystrophy (FSHD) is among the most common forms of muscular dystrophy. FSHD is caused by aberrant expression of the toxic DUX4 gene in muscle. Detecting endogenous DUX4 in patient tissue using conventional methods can be challenging, due to the low level of DUX4 expression. Therefore, developing simple and trustworthy DUX4 detection methods is an important need in the FSHD field. Here, we describe such a method, which uses the RNAscope assay, an RNA in situ hybridization (ISH) technology. We show that a custom-designed RNAscope assay can detect overexpressed DUX4 mRNA in transfected HEK293 cells and endogenous DUX4 mRNA in FSHD patientderived myotubes. The RNAscope assay was highly sensitive for tracking reductions in DUX4 mRNA following treatment with our therapeutic mi405 microRNA, suggesting that RNAscope-based DUX4 expression assays could be developed as a prospective outcome measure in therapy trials. This study could set the stage for optimizing and developing a new, rapid RNA ISH-based molecular diagnostic assay for future clinical use in the FSHD field.
\end{abstract}

Keywords: FSHD; DUX4; RNA in situ hybridization; RNA; RNAscope

\section{INTRODUCTION}

Facioscapulohumeral muscular dystrophy (FSHD) is among the most common types of muscular dystrophy with a prevalence of about 5-12 per 100,000 individuals (Deenen et al. 2014). FSHD shows autosomal dominant (FSHD1) or digenic (FSHD2) inheritance and the disease often manifests with slowly progressive muscle weakness involving the facial, scapular, upper arm, lower leg, and hip girdle muscles (Statland and Tawil 2014, 2016). Asymmetrical involvement can occur. Although the disease was named for the muscle groups most commonly affected, there is no universal pattern of muscle involvement and patients can show wide variability in symptoms. Clinical features often become evident in the second decade of life although age-at-onset and rate of progression may also vary (Statland and Tawil 2014, 2016).

FSHD is caused by aberrant expression of the double homeobox protein 4 (DUX4) gene (Lemmers et al. 2010, 2012). The DUX4 gene is located within repetitive elements, called D4Z4 repeats, located on the subtelomere of chromosome 4q35. Except in early embryogenesis or adult testis, this region is normally embedded in hetero-

Corresponding author: Scott.Harper@nationwidechildrens.org Article is online at http://www.rnajournal.org/cgi/doi/10.1261/rna. 070177.118. Freely available online through the RNA Open Access option. chromatin, and therefore DUX4 is not transcribed. In individuals with FSHD, the $4 q 35$ region is epigenetically derepressed (Lemmers et al. 2010, 2012). Several genetic conditions can trigger a change in the epigenetic structure of the 4q35 D4Z4 region from a heterochromatin state to a more open, euchromatin-like state. These derepressing epigenetic lesions permit DUX4 transcription, and if this occurs on a chromosome 4 allele containing an adjacent poly A signal for DUX4 (called the 4qA haplotype), the mRNA is polyadenylated and can be translated by the ribosome. The resultant DUX4 protein is toxic to muscle, cultured myocytes, and other nonmuscle cells in vitro (Lemmers et al. 2010, 2012; Wallace et al. 2011; Giesige et al. 2018).

Despite causing potentially devastating effects in muscles of FSHD patients, and increased cell death when expressed in vitro, DUX4 expression is relatively rare (Kowaljow et al. 2007; Snider et al. 2009, 2010; Wallace et al. 2011; Ferreboeuf et al. 2014). Indeed, DUX4 may typically be present in only a small percentage (0.1\%-0.01\%) of myonuclei from FSHD patient cell lines (Snider et al. 2010), and this relative scarcity has posed challenges for detecting DUX4 messenger RNA or DUX4 protein reliably using methods like $\mathrm{PCR}$, western blotting or

(C) 2019 Amini Chermahini et al. This article, published in RNA, is available under a Creative Commons License (Attribution 4.0 International), as described at http://creativecommons.org/licenses/by/4.0/. 
immunohistochemistry in vivo. For example, some PCR assays have required very large amounts of starting material and/or 55-70 PCR cycles to detect reverse-transcribed DUX4 expression from FSHD patient materials (Dixit et al. 2007; Jones et al. 2012). At the protein level, the currently available DUX4-specific antibodies have so far not been useful for reliably detecting endogenous DUX4 protein by western blot or immunofluorescence staining in patient biopsy material. In addition to impacting basic research, the difficulty to reliably detect DUX4 expression could pose challenging for future prospective clinical trials involving DUX4 inhibition therapies, where DUX4 levels would be useful as a therapeutic outcome measure.

As an alternative approach to detect DUX4 expression, here we tested a custom in situ hybridization (ISH) method using a powerful and highly specific technology called RNAscope (Wang et al. 2012). RNAscope relies upon "double Z" probe technology and specific signal amplification steps that virtually eliminate the background noise often encountered with traditional ISH approaches (Wang et al. 2012; Deleage et al. 2018). As a result, the RNAscope signal amplification method permits sensitive detection of low abundance RNAs, while also allowing localization of mRNAs in individual cells (Wang et al. 2012; Deleage et al. 2018). Because DUX4 has low abundance and sporadic expression, we reasoned that RNAscope could be an ideal method for DUX4 detection. At the time we began this study, the vendor (ACDBio) had not developed DUX4-targeted RNAscope probes and we therefore initiated custom designed DUX4 RNAscope probes to detect overexpressed and endogenous DUX4 mRNAs in vitro. These probes are now available as catalog items at ACDBio. The entire protocol from cell fixation to imaging takes about $8 \mathrm{~h}$ and can be accomplished during $1 \mathrm{~d}$. Our results demonstrate proof-of-principle for using RNAscope ISH technology to detect DUX4 mRNA at overexpressed and endogenous levels of expression in human cells, and set the stage for translating the method for detecting DUX4 in human FSHD biopsies.

\section{RESULTS}

\section{Detection of overexpressed DUX4 in transfected human HEK293 cells}

Our goal was to develop a novel and efficient technique for detecting DUX4 mRNA in mammalian cells. To do this, we tested the ability of custom-made DUX4-targeted RNAscope probes to detect overexpressed DUX4 mRNA in HEK293 cells transfected with a CMV.DUX4 expression plasmid. Sixteen hours later, we then fixed cells and stained with the RNAscope assay, using a diaminobenzidine (DAB) reagent which stains hybridized target mRNAs brown. We found that DUX4-transfected cells showed abundant, punctate brown dots and also some spider-like projections that were evident throughout the slides (Fig. 1). We detected no DUX4 signal in untransfected HEK293s (Fig. 1). As a positive control for the RNAscope assay, we used a probe targeting the housekeeping gene PPIB (peptidylprolyl isomerase $B$ ), which was provided with the RNAscope assay kit. Importantly, to demonstrate the specificity of our custom DUX4 RNAscope probes to detect DUX4 mRNA, we cotransfected HEK293 cells with the CMV.DUX4 expression plasmid and an U6 promoter-driven microRNA, called mi405, which we have previously shown significantly knocks down DUX4 mRNA using an RNA interference (RNAi) mechanism (Wallace et al. 2012, 2018). DUX4-expressing cells transfected with U6.mi405 showed microRNA-dose dependent reduction in the amount and intensity of brown punctate staining, thereby supporting the specificity of the DUX4 probe (Fig. 1). In addition, consistent with our prior work, qualitatively we observed that increasing amounts of the DUX4-targeted microRNA protected HEK293 cells from DUX4-dependent cell death, in a dose-dependent fashion (Fig. 1). Upon quantification with trypan blue cell viability assay, we found that mi405 coexpression produced dose-dependent protection from DUX4-induced cell death (Fig. 1).

\section{Detection of endogenous DUX4 in myotubes derived from FSHD patient biopsies}

We next determined the sensitivity of the RNAscope assay to detect endogenous DUX4 in FSHD patient cells. To do this, we differentiated the FSHD-affected 15A cell line and an unaffected paired control (15V) for $4 \mathrm{~d}$, and then performed RNAscope assay on fixed myotubes (Jones et al. 2012). We found abundant punctate brown staining in the $15 \mathrm{~A}$ cells, and absent or very faint background staining in unaffected $15 \mathrm{~V}$ myotubes (Fig. 2). We confirmed this staining pattern in four other cell lines derived from FSHD patient biopsies or unaffected family members (affected 16A and 17A; unaffected 16U and 17U) (Supplemental Fig. 1). Specifically, like the 15A-15U pairs, DUX4 RNAscope signal was detected in FSHD-affected cells (16A and 17A) but absent or at background levels in unaffected cells (16U and 17U). PPIB stain was again used as a positive control for the assay. To determine specificity of the RNAscope assay to detect endogenous DUX4, we electroporated cells with the U6.mi405 plasmid prior to differentiation, and then performed RNAscope staining. We found that mi405 treatment reduced RNAscope signals in differentiated myoblast cells, suggesting that the method was both sensitive and specific for detecting endogenous DUX4 mRNA (Figs. 2, 3).

We next quantified the DUX4 RNAscope signals in 15A and $15 \mathrm{U}$ cells using a microscopic morphometry method. Specifically, we developed a grid system and took digital photomicrographs of 20 microscopic fields within five 

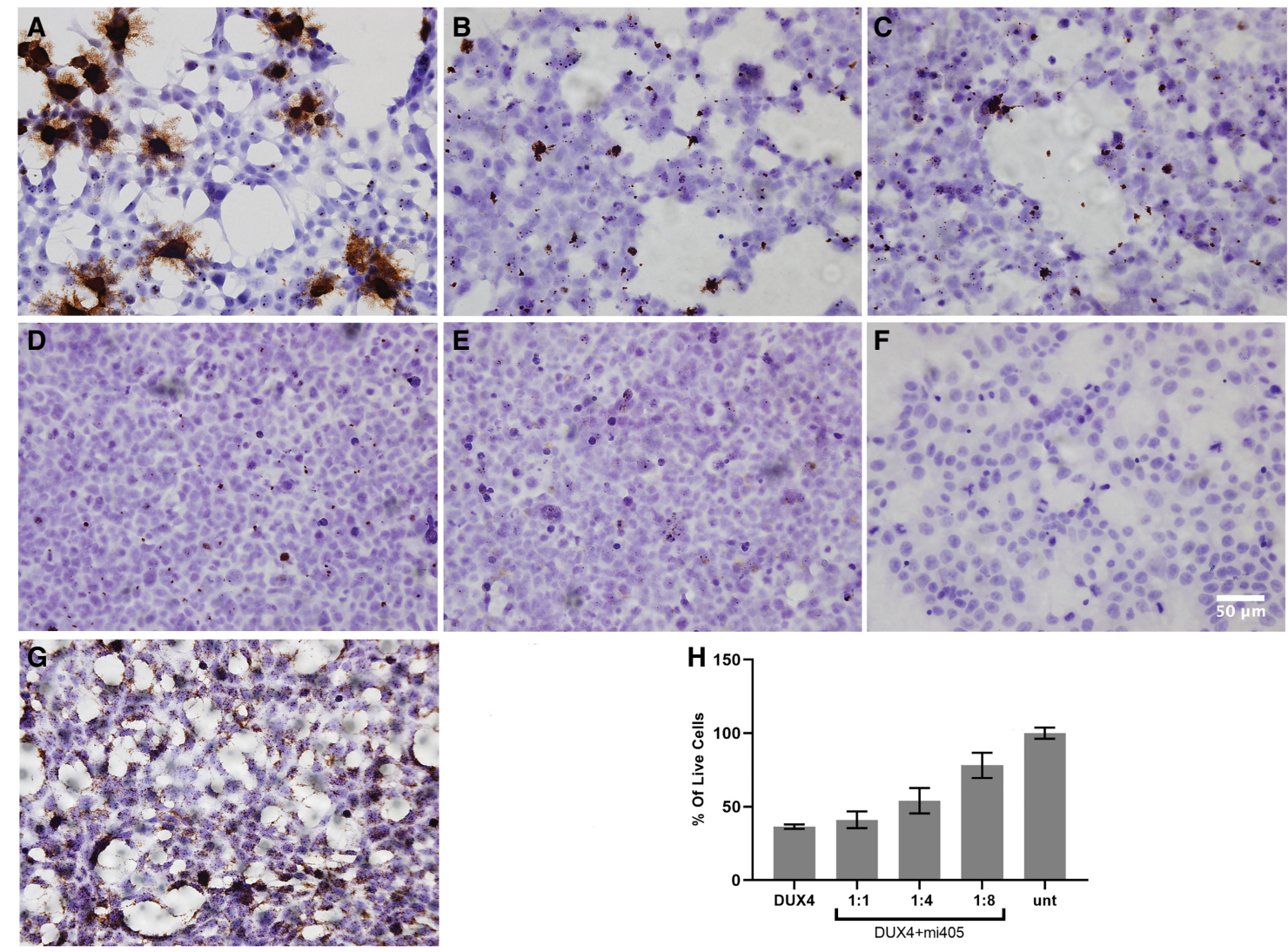

FIGURE 1. RNAscope specifically detected overexpressed DUX4 mRNA in transfected HEK293 cells. (A) DUX4-transfected cells showed punctate brown staining with RNAscope assay, while controls did not. Decreased brown staining with increased doses of DUX4-targeted microRNAs (mi405) demonstrated specificity. (B-D) DUX4 signal after cotransfection of HEK293 cells with CMV.DUX4 and U6.mi405 plasmids at 1:2, 1:4, and 1:8 ratios. (E) Absence of brown DUX4 signal in untransfected HEK293 cell line. (F) RNAscope negative control stain. (G) Housekeeping gene PPIB was detected in all HEK293 cells and served as a positive control for the assay. $(H)$ Cell viability assay demonstrated that mi405 reduced the levels of overexpressed DUX4 mRNA, and significantly protected cells from death at 1:4 and 1:8 weight ratios $(N=3$ independent experiments performed in triplicate); $P<0.0001$, ANOVA. $40 \times$ objective. Scale bar, 50 microns.

zones for every cell culture sample (Fig. 3A). We then measured DUX4 signal using Fiji/lmageJ software quantification (Wayne Rasband, National Institutes of Health) essentially following manufacturer's instructions (ACDBio), and generated a video example of the quantification method (Supplemental Fig. 2). We found that DUX4 signal was significantly higher in $15 \mathrm{~A}$ cells compared to $15 \mathrm{~V}$, where it was virtually absent, which was consistent with a previous study using other methods of detection (Jones et al. 2012). Despite increased DUX4 signal in $15 \mathrm{~A}$ compared to unaffected $15 \mathrm{~V}$ cells, only about $1 \%$ of the total area of 15A myotubes showed DUX4 RNAscope signal (Fig. 3B). This sporadic expression in only a small percentage of cells was also consistent with previous work (Snider et al. 2010). Nevertheless, in those rare cells where DUX4 was expressed, signals were often very high (Fig. 3C). To illustrate this, we quantified the number of DUX4-positive punctate foci, where one focus was defined as 16 pixels, as this was the smallest discernible RNAscope signal above background. Using this method, we found that some fields showed almost no signal, while others had thousands of DUX4+ foci per field (Fig. 3C). To confirm the reproducibility of this quantification method, a second person independently counted the same microscopic fields using the same methodology but with a blinded approach. Specifically, the second operator was provided individual images randomly and blinded across all groups. Importantly, the results were concordant between the first and second quantification (Fig. 3B,C; Supplemental Fig. 3). As a confirmation of signal specificity, DUX4 signal was significantly reduced to background in mi405-transfected 15A cells (Figs. 2, 3B,C). These data were consistent with our previous data showing that the DUX4-targeted mi405 construct significantly knocked down overexpressed DUX4 mRNA in human cells and in a DUX4 mouse model (Wallace et al. 2012, 2018). As a final confirmation of DUX4 expression in affected and unaffected cell lines as well as the impacts of DUX4 knockdown by miDUX4.405, we used quantitative RT-PCR to measure expression of the DUX4-activated human biomarkers 

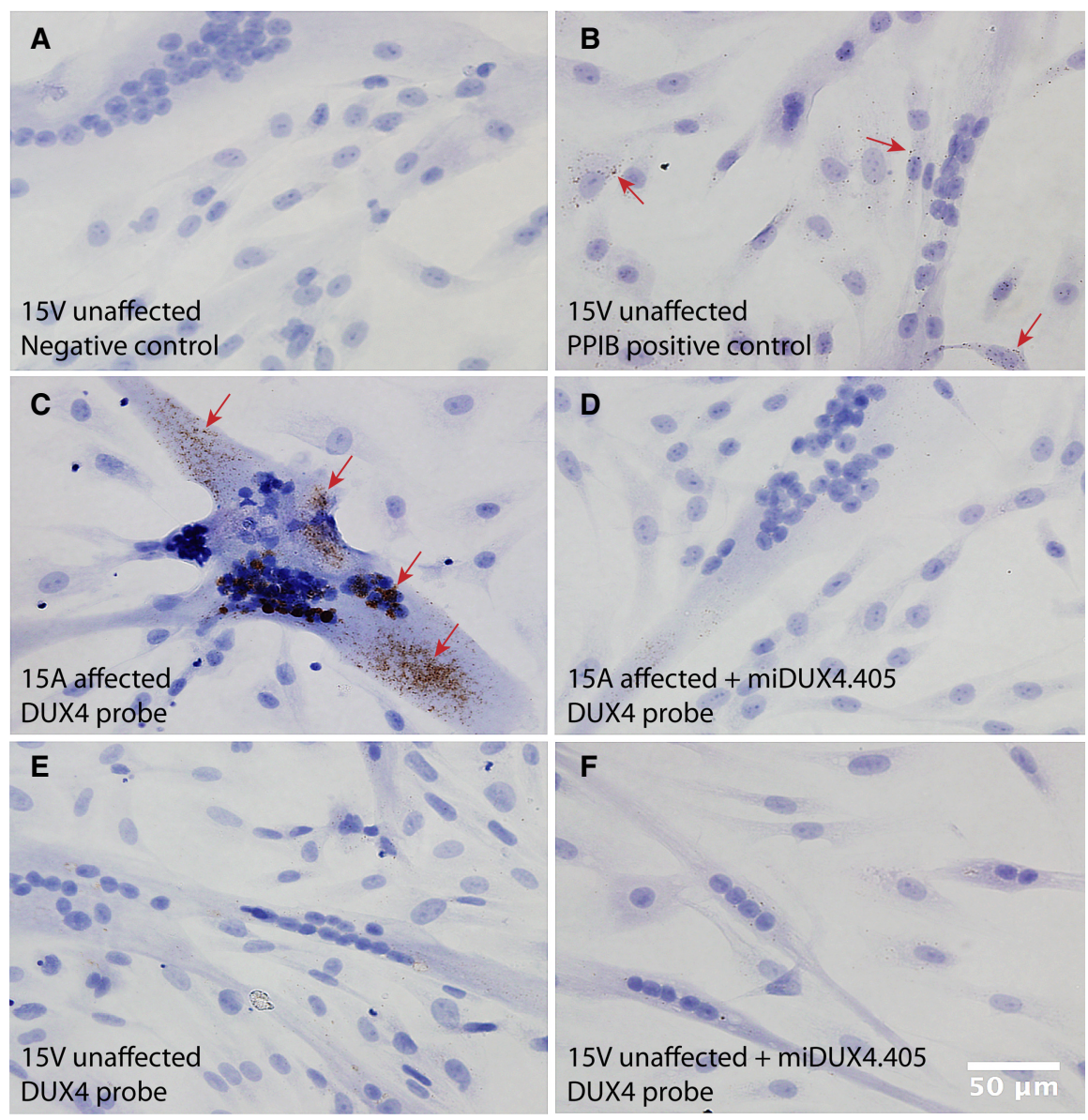

FIGURE 2. RNAscope detects endogenous DUX4 mRNA in 15A FSHD myotubes. FSHD 15A myotubes demonstrated higher amounts of DUX4 mRNA compared to non-FSHD 15V myotubes, as determined by RNAscope staining. Arrows indicate brown punctate signal. (A) Negative control stain. (B) 15V cell line stained with the housekeeping gene PPIB served as a positive control for the assay. (C) DUX4 expression in FSHD 15A myotubes was reduced or absent in (D) 15A cells transfected with U6.mi405 microRNAs. (E) Very weak or absent signal was present in the unaffected $15 \mathrm{~V}$ cell line alone and in $(F) 15 \mathrm{~V}$ transfected with U6.mi405 plasmid.

PRAMEF12 and MBD3L2 in cultured myotubes. We found that both biomarkers were present in affected cells (15A, 16A, 17A) but absent or extremely low in unaffected cells $(15 \mathrm{~V}, 16 \mathrm{U}, 17 \mathrm{U})$, and that affected cells treated with mi405 showed significantly reduced or absent DUX4 biomarker expression (Fig. 3D; Supplemental Fig. 4).

\section{DISCUSSION}

The FSHD field has made tremendous progress during the last decade, including the publication of a unified model of pathogenesis, development of numerous animal and cell models, and the emergence of several new promising therapeutic strategies. As a sign that the FSHD field has turned a corner toward translational work, there are now several efforts to establish clinical outcome measures for emerging therapeutic studies. Unfortunately, to our knowledge, at this time there are not established methods for reliably detecting endogenous DUX4 expression in clinical samples. As a result, it is currently infeasible to use DUX4 expression as a clinical biomarker for FSHD, although there has been considerable effort to identify and detect DUX4-associated biomarkers for use as an indirect indication of DUX4 gene expression (Yao et al. 2014; Rickard et al. 2015; Eidahl et al. 2016; Jagannathan et al. 2016; Heuvel et al. 2018). Although these will likely prove very useful, we propose that having the ability to directly measure DUX4 expression would be optimal for FSHD therapeutic strategies that are aimed at reducing DUX4 levels, such as an RNAi-based gene therapy we are working to translate (Wallace et al. 2012, 2018). Several approaches for DUX4 detection have been used in recent years. As mentioned, this is difficult due to the scarcity of DUX4 expression, even in FSHD patient cells and tissues. Although no published work has shown DUX4 protein detection in FSHD biopsies, it can be found in rare nuclei (generally $<1 \%$ ) of differentiated myocytes isolated from FSHD patients using indirect immunofluorescence staining with DUX4 antibodies. This scarcity and nonuniformity in expression within a single culture plate extends to DUX4 
A
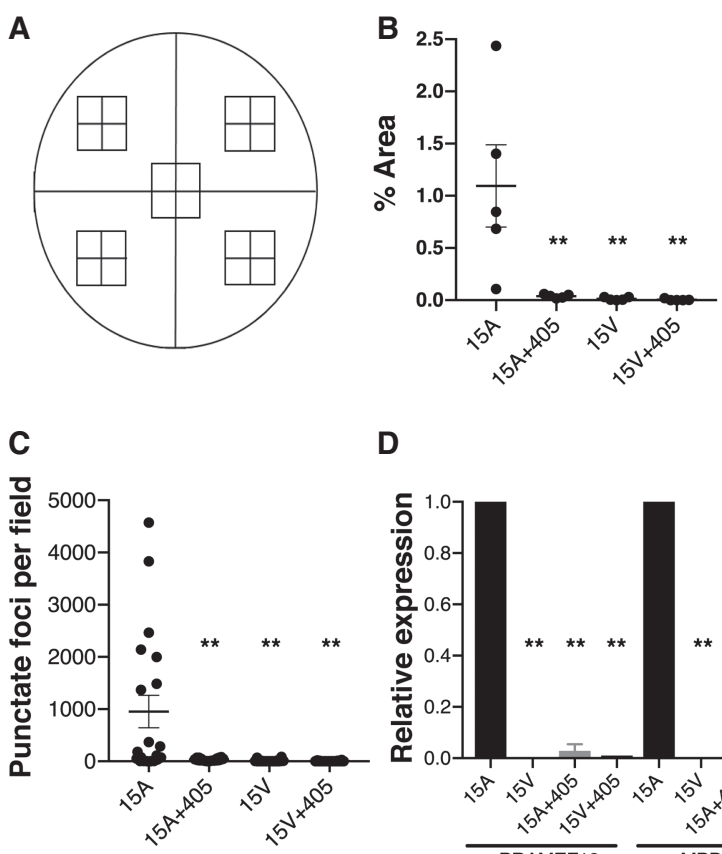

D

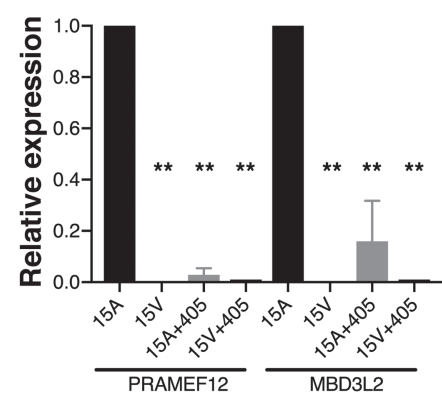

FIGURE 3. Quantification of DUX4 RNAscope signal and DUX4-activated biomarkers. (A) Schematic representation of a cover slip on which FSHD and control myoblasts were grown and differentiated to myotubes. For each sample, a total of 20 image fields were taken from five zones (four quadrants and one central zone). DUX4 RNAscope signal was quantified as demonstrated in the video in Supplemental Figure 2. (B) Percent area of DUX4 signal in one representative experiment (from three independent experiments). Each data point represents total \% area per zone. In this experiment, DUX4 signal was significantly elevated in 15A myotubes compared to unaffected $15 \mathrm{~V}$ controls and to $15 \mathrm{~A}$ cells transfected with the mi405 therapeutic microRNA. $\left(^{* *}\right)$ Represents significant differences from untreated 15A FSHD cells ( $P<0.01$; ANOVA). (C) Data presented here indicate that despite low abundance relative to the entire $15 \mathrm{~A}$ culture, the $\sim 1 \%$ of cells showing DUX4 signal often expressed high amounts of DUX4 mRNA. Here, each data point represents the number of DUX4 positive foci per field in a representative culture. One DUX4 focus was defined as 16 pixels, which was the minimum visible DUX4 signal. Again, DUX4 signal was absent or very low in unaffected $15 \mathrm{~V}$ cells, as well as affected $15 \mathrm{~A}$ cells transfected with mi 405 plasmid. $\left.{ }^{* *}\right)$ Represents significant differences from untreated 15A myotubes $(P<0.01$; ANOVA). (D) QPCR assays of DUX4-activated biomarkers, PRAMEF12 and MBD3L2, in 15A and 15V cells. Biomarker expression was used to confirm the specificity of the RNAscope assay and knockdown of endogenous DUX4 by miDUX4.405. Both biomarkers were significantly elevated in affected 15A myotubes, but absent or virtually absent in $15 \mathrm{~V}$ cells (no signal at cycle 40). PRAMEF12 and MBD3L2 were significantly reduced in 15A cells transfected with miDUX4.405 plasmid. Data were acquired from $N=3$ independent experiments, with each QPCR assay performed in triplicate. $\left.{ }^{* *}\right)$ Represents significant differences from untreated $15 \mathrm{~A}$ myotubes $(P<0.01$; ANOVA).

mRNA, where again DUX4 is absent in most cells (Heuvel et al. 2018). This phenomenon makes a global transcript detection method like QPCR challenging, as it would not be able to indicate how many cells on the plate were expressing DUX4, and there would be a dilution effect on
DUX4 signal in a total RNA population since most cells in the culture would not express it. It may therefore be preferable to have the ability to detect DUX4 mRNA and-importantly for therapy studies-accurately quantify DUX4 levels in single cells. This could be done using complex cutting edge methods like single-cell RNA sequencing (Heuvel et al. 2018), or ISH, which is what we developed here.

We note that DUX4 was detected by traditional immunofluorescence-based ISH in one other study, but that single paper was not focused on assay development, and the usefulness of ISH for broad application and quantification was not determined (Ferreboeuf et al. 2014). Specifically, DUX4 signal was shown only for three nuclei at high power, and no quantification or validation of specificity were pursued. In contrast, we extensively characterized the specificity of a new DUX4-targeted RNAscope method here, and described in-depth a strategy for quantification using light microscopy and publicly available ImageJ software. Specifically, as a first step to develop a new assay for DUX4 detection, we tested custom-made (but now available) RNAscope probes to detect overexpressed DUX4 in transfected HEK293s, and importantly, endogenous DUX4 transcripts in FSHD patient-derived myoblasts. Our results in HEK293 cells, which do not naturally express DUX4, demonstrated that the assay was able to detect DUX4 with high specificity. In particular, we observed DUX4 mRNA signal only in cells transfected with CMV.DUX4 plasmid transfection, and did not detect signal in untransfected cells. Importantly, as another indication of specificity, we also found that DUX4 signal decreased with increasing doses of our therapeutic miDUX4.405 construct, and that this reduced DUX4 signal correlated with increased protection from cell death, compared to DUX4-only controls. We also showed sensitivity of the assay to detect comparatively low levels of endogenous DUX4 transcript in patient-derived myotubes, and again confirmed probe specificity in these cells as demonstrated by reduced DUX4 signal, as well as DUX4-activated biomarkers (MBD3L2 and PRAMEF12) in FSHD patient cells transfected with U6.miDUX4.405 plasmids. Finally, we used a blinded approach to support that the quantification method was reproducible among two different operators. Our future work with this assay will focus on detecting DUX4 in vivo, using muscle sections from an FSHD mouse model and biopsies from human patients.

In conclusion, we found that RNAscope is a highly sensitive method for detecting DUX4 mRNA in vitro, and may enable us to develop a new, rapid RNA ISH-based molecular diagnostic assay for FSHD. Importantly, the DUX4 RNAscope assay was able to detect reductions in DUX4 mRNA following treatment with our therapeutic microRNA, miDUX4.405. These results suggested that RNAscope may be developed for use as a clinical outcome measure after DUX4-modulating treatments. 


\section{MATERIALS AND METHODS}

\section{Transfection of DUX4 and miDUX4.405 expression plasmids into human HEK293 cells}

HEK293 cells were seeded in triplicate on coverslips in a 24-well plate at a density of 200,000 cells per well. Cells were incubated in Dulbecco's modified eagle medium (DMEM) (Lonza) supplemented with $10 \%$ fetal bovine serum (Hyclone), L-glutamine (Invitrogen) and penicillin/streptomycin (Sigma-Aldrich) at $37^{\circ} \mathrm{C}$ in $5 \% \mathrm{CO}_{2}$. Upon reaching $70 \%$ confluency, cells were transfected with $500 \mathrm{ng}$ of CMV.DUX4 expression plasmid using Lipofectamine-2000 (Thermo Fisher Scientific), according to manufacturer's instructions. Sixteen hours after transfection, cells were fixed with 4\% PFA and RNAscope staining was performed following manufacturer's instructions (detailed below). To demonstrate specificity, we performed a DUX4 dose-response in which DUX4 was knocked down with our lead therapeutic DUX4-targeted microRNA, called mi405. For those experiments, cells were cotransfected with CMV.DUX4 and U6.mi405 expression plasmids at 1:1, 1:4, and 1:8 weight ratios (Wallace et al. 2012, 2018).

\section{Culturing of FSHD patient myotubes}

We used previously described FSHD patient myotubes to test the ability of our custom RNAscope assay to detect endogenous DUX4 (Jones et al. 2012). Human immortalized myoblasts 15V, primary $16 \mathrm{U}$ and $17 \mathrm{U}$ (unaffected) and immortalized 15A, primary $16 \mathrm{~A}$ and 17A (FSHD affected) were seeded with 4:1 ratio of DMEM: Medium 199 supplemented with 15\% fetal bovine serum (Hyclone), $30 \mathrm{ng} / \mathrm{mL}$ zinc sulfate (Fisher Scientific), $1.4 \mu \mathrm{g} / \mathrm{mL}$ Vitamin B12 (Sigma-Aldrich), $55 \mathrm{ng} / \mathrm{mL}$ dexamethasone (Sigma-Aldrich), $2.5 \mathrm{ng} / \mathrm{mL}$ human growth factor (Chemicon International), $10 \mathrm{ng} / \mathrm{mL}$ fibroblast growth factor (BioPioneer HRP) and $20 \mathrm{mM}$ HEPES (Sigma-Aldrich). For elucidating specificity of the DUX4 RNAscope probe, $15 \mathrm{~V}$ and $15 \mathrm{~A}$ myoblasts were transfected with U6.mi405 plasmid via electroporation (Lonza) and then $24 \mathrm{~h}$ later, switched to differentiation media (4:1 ratio of DMEM:Medium 199 supplemented with 15\% KOSR, 2 mM L-glutamine, 1\% antibiotics/ antimycobiotics [cat. no. 15-240-096 Gibco], 1 mM sodium pyruvate, $20 \mathrm{mM}$ HEPES buffer) for up to $7 \mathrm{~d}$.

\section{RNAscope staining}

Cells were grown on cover slips, which were then mounted on microscopy slides for RNAscope assay. Cells were washed twice with PBS, then fixed with 4\% PFA (Fisher Scientific) for 30 min at room temperature, and finally dehydrated with $50 \%, 70 \%$, and $100 \%$ ethyl alcohol gradients for $5 \mathrm{~min}$ each at room temperature. Cells were then rehydrated with $70 \%$ and $50 \%$ ethyl alcohol gradients for 2 min each and finally treated with PBS for $10 \mathrm{~min}$, followed by hydrogen peroxide (cat. no. 322335 ACDBio) and protease III (cat. no. 322337 ACDBio) at room temperature for $10 \mathrm{~min}$ each, and then washed with PBS. Probes were then added for $2 \mathrm{~h}$ at $40^{\circ} \mathrm{C}$ within a humidity control chamber. Prior to initiating this study, a DUX4 probe did not exist, and we worked with ACDBio to have one custom developed. It is now listed in the ACDBio catalog as Hs-DUX4-No-XMm, NM_001306068.2, target region: 36-1689 (cat. no. 498541). Signal amplification and detection reagents (cat. no. 322310 ACDBio) were applied sequentially and incubated in AMP 1, AMP 2, AMP 3, AMP 4, AMP 5, and AMP 6 reagents, for 30, 15, 30, 15, 30, 15 min, respectively. Before adding each AMP reagent, samples were washed twice with washing buffer (cat. no. 310091 ACDBio). The samples were then counterstained with $50 \%$ Gill's hematoxylin I (cat. no. HXGHE1LT, American Master Tech Scientific) for $2 \mathrm{~min}$ at room temperature, rinsed with tap water, placed in $0.02 \%$ ammonia water, followed by another tap water rinse. Samples were then dehydrated with $70 \%$ and $100 \%$ ethyl alcohol gradients for $2 \mathrm{~min}$ each, followed by xylene treatment for $5 \mathrm{~min}$ (cat. no. X3P-1GAL, Fisher Scientific). Mounting media and cover slips were then added to slides for imaging. Images were captured using an Olympus DP71 microscope.

\section{RNAscope quantification}

For quantification, 20 images per sample were taken from a total of five zones (four quadrants and a central zone). Four 100× images were taken from a random area within each zone. DUX4 RNAscope signals were quantified using ImageJ (Fiji) software as directed by the vendor's guidelines (TS 46-003/Rev A/Date 6212018). Detailed quantification procedures are shown in the Supplemental Video. The amount of the detected signal compared to the total area of each image was calculated using Image $\mathrm{J}$ and plotted as both percent area and pixel units, where 16 pixels was considered equivalent to one DUX4 mRNA focus.

\section{SUPPLEMENTAL MATERIAL}

Supplemental material is available for this article.

\section{ACKNOWLEDGMENTS}

This work, including partial salaries of the coauthors, was supported in part by grants from the National Institute of Neurological Disorders and Stroke (NINDS) Translational Program grant 1R21NS101166 (S.Q.H.), the National Institute of Arthritis, Musculoskeletal and Skin Diseases NIAMS Wellstone Center at the University of Massachusetts (2 P50 HD060848-12), the NIAMS Center of Research Translation in Muscular Dystrophy Therapeutic Development grant at Nationwide Children's Hospital (1P50AR070604; S.Q.H.), the Muscular Dystrophy Association (S.Q.H.) and the Chris Carrino Foundation (S.Q.H.). During the course of this study, Dr. Rashnonejad's salary was supported by a postdoctoral fellowship from the Friends of FSH Research Foundation. We thank Dr. Zarife Sahenk for advice regarding morphometric quantification of myotubes.

Received December 21, 2018; accepted June 7, 2019.

\section{REFERENCES}

Deenen JC, Arnts $H$, van der Maarel SM, Padberg GW, Verschuuren JJ, Bakker E, Weinreich SS, Verbeek AL, van Engelen BG. 2014. Population-based incidence and prevalence of facioscapulohumeral dystrophy. Neurology 83: 1056-1059. doi:10.1212/WNL.0000000000000797 
Deleage C, Chan CN, Busman-Sahay K, Estes JD. 2018. Next-generation in situ hybridization approaches to define and quantify HIV and SIV reservoirs in tissue microenvironments. Retrovirology 15: 4. doi:10.1186/s12977-017-0387-9

Dixit M, Ansseau E, Tassin A, Winokur S, Shi R, Qian H, Sauvage S, Mattéotti C, van Acker AM, Leo O, et al. 2007. DUX4, a candidate gene of facioscapulohumeral muscular dystrophy, encodes a transcriptional activator of PITX1. Proc Natl Acad Sci 104: 1815718162. doi:10.1073/pnas.0708659104

Eidahl JO, Giesige CR, Domire JS, Wallace LM, Fowler AM, Guckes SM, Garwick-Coppens SE, Labhart P, Harper SQ. 2016. Mouse Dux is myotoxic and shares partial functional homology with its human paralog DUX4. Hum Mol Genet 25: 4577-4589. doi:10.1093/hmg/ddw287

Ferreboeuf M, Mariot V, Furling D, Butler-Browne G, Mouly V, Dumonceaux J. 2014. Nuclear protein spreading: implication for pathophysiology of neuromuscular diseases. Hum Mol Genet 23: 4125-4133. doi:10.1093/hmg/ddu129

Giesige CR, Wallace LM, Heller KN, Eidahl JO, Saad NY, Fowler AM, Pyne NK, Al-Kharsan M, Rashnonejad A, Amini-Chermahini G, et al. 2018. AAV-mediated follistatin gene therapy improves functional outcomes in the TIC-DUX4 mouse model of FSHD. JCl Insight 3: 123538. doi:10.1172/jci.insight.123538

Heuvel AVD, Mahfouz A, Kloet SL, Balog J, Engelen BGMV, Tawil R, Tapscott SJ, Maarel SMV. 2018. Single-cell RNA-sequencing in facioscapulohumeral muscular dystrophy disease etiology and development. Hum Mol Genet 28: 1064-1075. doi:10.1093/hmg/ ddy 400

Jagannathan S, Shadle SC, Resnick R, Snider L, Tawil RN, van der Maarel SM, Bradley RK, Tapscott SJ. 2016. Model systems of DUX4 expression recapitulate the transcriptional profile of FSHD cells. Hum Mol Genet 25: 4419-4431. doi:10.1093/hmg/ddw271

Jones TI, Chen JC, Rahimov F, Homma S, Arashiro P, Beermann ML, King OD, Miller JB, Kunkel LM, Emerson CP, et al. 2012. Facioscapulohumeral muscular dystrophy family studies of DUX4 expression: evidence for disease modifiers and a quantitative model of pathogenesis. Hum Mol Genet 21: 4419-4430. doi:10 $.1093 / \mathrm{hmg} / \mathrm{dds} 284$

Kowaljow V, Marcowycz A, Ansseau E, Conde CB, Sauvage S, Mattéotti C, Arias C, Corona ED, Nuñez NG, Leo O, et al. 2007. The DUX4 gene at the FSHD1A locus encodes a pro-apoptotic protein. Neuromuscul Disord 17: 611-623. doi:10.1016/j.nmd .2007.04.002

Lemmers RJ, van der Vliet PJ, Klooster R, Sacconi S, Camaño P, Dauwerse JG, Snider L, Straasheijm KR, van Ommen GJ, Padberg GW, et al. 2010. A unifying genetic model for facioscapulohumeral muscular dystrophy. Science 329: 1650-1653. doi:10 $.1126 /$ science. 1189044
Lemmers RJ, Tawil R, Petek LM, Balog J, Block GJ, Santen GW Amell AM, van der Vliet PJ, Almomani R, Straasheijm KR, et al. 2012. Digenic inheritance of an SMCHD1 mutation and an FSHD-permissive D4Z4 allele causes facioscapulohumeral muscular dystrophy type 2. Nat Genet 44: 1370-1374. doi:10.1038/ng .2454

Rickard AM, Petek LM, Miller DG. 2015. Endogenous DUX4 expression in FSHD myotubes is sufficient to cause cell death and disrupts RNA splicing and cell migration pathways. Hum Mol Genet 24: 5901-5914. doi:10.1093/hmg/ddv315

Snider L, Asawachaicharn A, Tyler AE, Geng LN, Petek LM, Maves L, Miller DG, Lemmers RJ, Winokur ST, Tawil R, et al. 2009. RNA transcripts, miRNA-sized fragments and proteins produced from D4Z4 units: new candidates for the pathophysiology of facioscapulohumeral dystrophy. Hum Mol Genet 18: 2414-2430. doi:10 $.1093 / \mathrm{hmg} / \mathrm{ddp} 180$

Snider L, Geng LN, Lemmers RJ, Kyba M, Ware CB, Nelson AM, Tawil R, Filippova GN, van der Maarel SM, Tapscott SJ, et al. 2010. Facioscapulohumeral dystrophy: incomplete suppression of a retrotransposed gene. PLoS Genet 6: e1001181. doi:10 .1371/journal.pgen.1001181

Statland J, Tawil R. 2014. Facioscapulohumeral muscular dystrophy. Neurol Clin 32: 721-728. doi:10.1016/j.ncl.2014.04.003

Statland JM, Tawil R. 2016. Facioscapulohumeral muscular dystrophy. Continuum (Minneap Minn) 22: 1916-1931. doi:10.1097/WCO .0b013e32834959af

Wallace LM, Garwick SE, Mei W, Belayew A, Coppee F, Ladner KJ, Guttridge D, Yang J, Harper SQ. 2011. DUX4, a candidate gene for facioscapulohumeral muscular dystrophy, causes p53-dependent myopathy in vivo. Ann Neurol 69: 540-552. doi:10.1002/ ana. 22275

Wallace LM, Liu J, Domire JS, Garwick-Coppens SE, Guckes SM, Mendell JR, Flanigan KM, Harper SQ. 2012. RNA interference inhibits DUX4-induced muscle toxicity in vivo: implications for a targeted FSHD therapy. Mol Ther 20: 1417-1423. doi:10.1038/mt .2012 .68

Wallace LM, Saad NY, Pyne NK, Fowler AM, Eidahl JO, Domire JS, Griffin DA, Herman AC, Sahenk Z, Rodino-Klapac LR, et al. 2018. Pre-clinical safety and off-target studies to support translation of AAV-mediated RNAi therapy for FSHD. Mol Ther Methods Clin Dev 8: 121-130. doi:10.1016/j.omtm.2017.12.005

Wang F, Flanagan J, Su N, Wang LC, Bui S, Nielson A, Wu X, Vo HT, Ma XJ, Luo Y. 2012. RNAscope: a novel in situ RNA analysis platform for formalin-fixed, paraffin-embedded tissues. J Mol Diagn 14: 22-29. doi:10.1016/j.jmoldx.2011.08.002

Yao Z, Snider L, Balog J, Lemmers RJ, Van Der Maarel SM, Tawil R, Tapscott SJ. 2014. DUX4-induced gene expression is the major molecular signature in FSHD skeletal muscle. Hum Mol Genet 23: 5342-5352. doi:10.1093/hmg/ddu251 

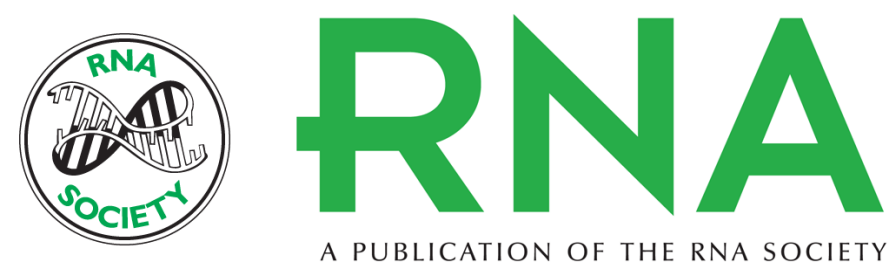

A PUBLICATION OF THE RNA SOCIETY

\section{RNAscope in situ hybridization-based method for detecting DUX4 RNA expression in vitro}

Gholamhossein Amini Chermahini, Afrooz Rashnonejad and Scott Q. Harper

RNA 2019 25: 1211-1217 originally published online June 17, 2019

Access the most recent version at doi:10.1261/rna.070177.118

\section{Supplemental http://rnajournal.cshlp.org/content/suppl/2019/06/17/rna.070177.118.DC1 \\ Material}

References This article cites 22 articles, 2 of which can be accessed free at: http://rnajournal.cshlp.org/content/25/9/1211.full.html\#ref-list-1

Open Access Freely available online through the RNA Open Access option.

Creative This article, published in $R N A$, is available under a Creative Commons License Commons (Attribution 4.0 International), as described at

License http://creativecommons.org/licenses/by/4.0/.

Email Alerting Receive free email alerts when new articles cite this article - sign up in the box at the Service top right corner of the article or click here.

To subscribe to $R N A$ go to:

http://rnajournal.cshlp.org/subscriptions 